\title{
Therapeutic Interventions in Adult Low-Grade Gliomas
}

\author{
Majid Chammas ${ }^{a}$ \\ Fadi Saadeh $^{\mathrm{a}}$ \\ Maya Maaliki $^{a}$ \\ Hazem Assi ${ }^{b}$ \\ ${ }^{a}$ American University of Beirut, \\ Faculty of Medicine, \\ Beirut, Lebanon \\ ${ }^{b}$ Department of Internal Medicine, \\ Naef K. Basile Cancer Institute, \\ American University \\ of Beirut Medical Center, \\ Beirut, Lebanon
}

Treating adult low-grade gliomas (LGGs) is particularly challenging due to the highly infiltrative nature of this type of brain cancer. Although surgery, radiotherapy, and chemotherapy are the mainstay treatment modalities for LGGs, the optimal combination management plan for a particular patient based on individual symptoms and the risk of treatment-induced toxicity remains unclear. This review highlights the competency and limitations of standard treatment options while providing an essential therapeutic update regarding current clinical trials aimed at implementing targeted therapies with morbidity rates lower than those for current LGG treatments and also augmenting the killing of cancerous cells while maintaining an improved quality of life.

Key Words low-grade glioma, chemotherapy, targeted therapy, brain cancer, radiotherapy, clinical trials.
Received May 8, 2018

Revised June 18, 2018

Accepted June 19, 2018

\section{Correspondence}

Hazem Assi, MD

Department of Internal Medicine,

Naef K. Basile Cancer Institute,

American University

of Beirut Medical Center,

P.O.Box 11-0236/Riad El-Solh,

Beirut 1107-2020, Lebanon

Tel +961 1350000

Fax +9611351706

E-mail1ha157@aub.edu.lb

\section{INTRODUCTION}

Low-grade gliomas (LGGs; WHO grade II) are primary slow-growing brain tumors derived from glial cells, namely astrocytes and oligodendroglia. These diffusely infiltrative and potentially malignant grade II gliomas include diffuse astrocytomas, pleomorphic xanthoastrocytomas, and oligodendrogliomas (ODs). Previously described mixed gliomas, namely oligoastrocytomas, are now classified as either astrocytoma or OD based on their histological and genetic distinctions. The wide diversity of these brain tumors necessitated an adjustment of the WHO classification in 2016, whereby both tumor phenotypes (e.g., growth patterns) and genotypes (e.g., distinct mutations) were used as grouping criteria. This allowed for the gliomas to be categorized based on both prognostic features as well as specific therapeutic modalities. Indeed, diffuse astrocytomas are now more closely nosologically related to ODs than to pilocytic astrocytomas, and this adjusted classification has important therapeutic implications in terms of tumor management. ${ }^{1}$

Recent characterizations of LGGs have shown that these tumors can be subclassified into three subtypes with distinct clinical behaviors based on the IDH1 and IDH2 mutations and the codeletion status of chromosomes 1p and 19q. LGGs with an IDH mutation either harbored a TP53 mutation or had 1p/19q codeletion. IDH-mutant LGGs with 1p/19q codeletion showed mutations in CIC, NOTCH1, FUBP1, and the TERT promoter, and had the most-favorable outcome clinically, whereas nearly all $I D H$ mutants without $1 \mathrm{p} / 19 \mathrm{q}$ codeletion had TP53 mutations (94\%) and ATRX inactivation (86\%). LGGs without an IDH mutation were similar to glioblastomas clinically and molecularly, and thus have had the least-favorable outcome. ${ }^{2}$

Around 2,000 to 3,000 cases of LGGs are diagnosed every year in the US, representing approximately $15 \%$ of all primary brain tumors. The prevalence of LGGs is higher in men and white people, and the incidence peaks between 35 and 44 years of age. ${ }^{3}$ Although low-

(a) This is an Open Access article distributed under the terms of the Creative Commons Attribution Non-Commercial License (https://creativecommons.org/licenses/by-nc/4.0) which permits unrestricted non-commercial use, distribution, and reproduction in any medium, provided the original work is properly cited. 
grade astrocytomas are more often diagnosed than ODs, the incidence rates of ODs are increasing. ${ }^{4}$ Malignant transformation of LGGs into high-grade gliomas (HGGs; grades III and IV) occurs in most patients, ultimately leading to death. HGG patients have a median survival of 1 to 2 years, whereas the median survival time for LGG patients is between 5 and 10 years. ${ }^{5}$ Regarding presentation, the most-common presenting symptom is a seizure, particularly in OD patients. ${ }^{6}$ Defining a management plan for patients with LGGs has caused controversy among neuro-oncologists, mainly due to a lack of sufficient clinical trials. Surgical resection, radiotherapy, and chemotherapy are generally the main treatment options (Fig. 1, Table 1).

\section{TREATMENT MODALITIES}

\section{Surgery}

The diffusely infiltrative nature of LGGs poses a critical challenge to the ability of neurosurgeons to perform gross total tumor resection. Overcoming this challenge would have major prognostic value, especially since the most-important independent spontaneous factor for a poor prognosis is a large tumor, and larger tumors tend to expand into more-eloquent areas of the cortex, thus hindering their gross total resection. ${ }^{7}$ Despite the relatively high risk of incomplete resection, surgery remains a significant first-line treatment option, and the extent of resection (EOR) strongly correlates with overall survival (OS). Smith et al. ${ }^{8}$ confirmed this in a postresection volumetric analysis of residual tumors in LGG patients, in that the 5- and 8-year OS rates were $97 \%$ and $91 \%$, respectively, for an

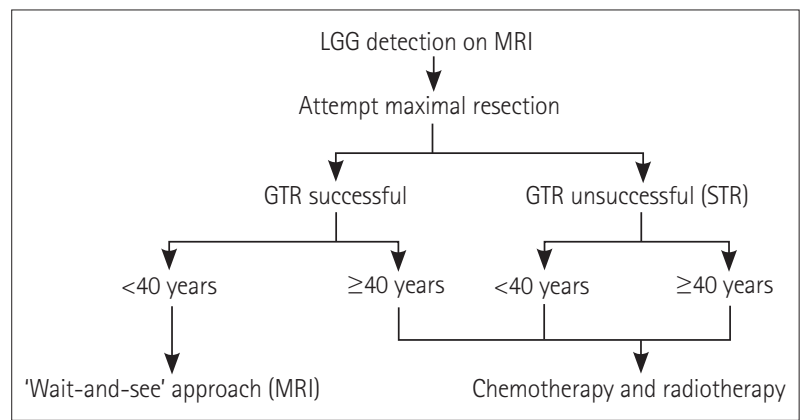

Fig. 1. General treatment algorithm for LGG. Low-risk patients are those with successful GTR and age $<40$ years. High-risk patients are those with either STR or age $\geq 40$ years. GTR: gross total resection, LGG: low-grade glioma, STR: subtotal resection.

Table 1. Summary of treatment modalities in low-grade glioma categorized according to the studied variables

\begin{tabular}{|c|c|c|c|c|}
\hline Treatment modality & Variable & & & References \\
\hline \multirow[t]{3}{*}{ Surgery } & EOR & 5 -year OS rate* & & 39,27 \\
\hline & $\geq 90 \%$ & $91-97 \%$ & & \\
\hline & $<90 \%$ & $60-76 \%$ & & \\
\hline \multirow[t]{10}{*}{ Radiotherapy } & Risk level & & Management & 31 \\
\hline & Low & IDHmut & 'Wait and see' & \\
\hline & & IDHwt & Radiochemotherapy & \\
\hline & High & - & Radiochemotherapy & \\
\hline & Dose & 5-year OS rate & & \\
\hline & 45 vs. 59.4 Gy & $58 \%$ vs. $59 \%$ & & 20 \\
\hline & 50.4 vs. $64.8 \mathrm{~Gy}$ & $72 \%$ vs. $64 \%$ & & 36 \\
\hline & Timing & PFS* $^{*}$ & OS & \\
\hline & Early vs. late (median time) & 5.3 vs. 3.4 years & 7.4 vs. 7.2 years & 3 \\
\hline & Early vs. late (5-year rate) & $44 \%$ vs. $37 \%$ & $63 \%$ vs. $66 \%$ & 21 \\
\hline \multirow[t]{10}{*}{ Chemotherapy } & Regimen & OS* & & \\
\hline & PCV+radiotherapy vs. radiotherapy & 13.3 vs. 7.8 years & & 7 \\
\hline & TMZ+radiotherapy vs. radiotherapy & $65 \%$ vs. $54 \%$ (3-year rate) & & 17 \\
\hline & Postoperative TMZ alone & $82 \%$ (3-year rate) & & 5 \\
\hline & & & 4-year PFS ${ }^{+}$ & \\
\hline & TMZ vs. radiotherapy & Overall & $3.3 \%$ vs. $3.8 \%$ & 1 \\
\hline & & Molecular subtype & & \\
\hline & & IDH mutant without codeletion & 36 vs. 55 months & \\
\hline & & IDH mutant with codeletion & n.s. & \\
\hline & & IDH wild-type & n.s. & \\
\hline
\end{tabular}

*Statistically significant, ${ }^{+}$OS could not be determined.

EOR: extent of resection, n.s.: not significant, OS: overall survival, PCV: procarbazine, lomustine, and vincristine, PFS: progression-free survival, TMZ: temozolomide. 
EOR of at least $90 \%$, and $76 \%$ and $60 \%$ for an EOR of less than $90 \%$. It was therefore concluded that a more-extensive resection resulted in a more-favorable prognosis. Another interesting retrospective cohort study examined the benefits of gross total resection (GTR) versus near-total resection (NTR) and subtotal resection (STR). FLAIR imaging was used to assess the tumor volume preoperatively and postoperatively to determine the EOR. The obtained results validated the preference to use GTR over NTR and STR, since the 5-year OS rates of these procedures were $95 \%, 80 \%$, and $70 \%$, respectively. These proportions demonstrate that when the ability to perform GTR is hindered by the tumor being located in eloquent cortical regions, then NTR and STR could still offer some survival advantage. The progression-free survival (PFS) rate and the malignant degeneration-free survival rate (based on the increased median time to convert to a HGG) were also higher in GTR than NTR and STR. GTR should therefore always be performed whenever safely permitted, with eloquent cortical zone preservation constituting a limitation to such gross total resection necessitating attempting NTR or STR. ${ }^{9}$

Recent studies have attempted to identify molecular markers in LGGs that are correlated with a favorable postresection prognosis. In a large molecularly defined cohort study, in which molecular genetic examinations were applied to LGG tumors after resection (IDH1 mutation, 1p/19q codeletion, and p53 mutation), Cordier et al. ${ }^{10}$ showed that the association between a better prognosis and a higher EOR did not correlate with a more-favorable genetic profile (e.g., IDH mutation). This essentially means that the better prognosis was due to the high EOR of the tumor rather than to a more-favorable genetic profile. Therefore, a high EOR could benefit the prognosis even with LGGs lacking good prognostic genetic markers such as $1 \mathrm{p} / 19 \mathrm{q}$ codeletion, and should always be attempted to increase OS. Wijnenga et al. ${ }^{11}$ studied postoperative tumor volumes in patients after correcting for molecular profiles, such as IDH mutation, based on the 2016 WHO classification of LGGs. Their results verified the significance of gross total resection as the first-line treatment for molecularly defined LGGs, as highlighted by even minor postoperative residues in $I D H$-mutated astrocytoma negatively influencing OS. It is therefore imperative to look for and safely remove any tumor remnants regardless of their volumetric extent, especially in IDH-mutant astrocytoma.

Many studies have also investigated the potential benefit of using intraoperative MRI (iMRI) scanning to maximize the EOR. Nimsky et al. ${ }^{12}$ found that iMRI increased the EOR in seven out of nine patients undergoing surgery; the other two had remnants in extremely eloquent areas that hindered their resection. This finding is supported by another study showing that tumor resections performed with iMRI guidance produced improved OS rates compared to national databases. ${ }^{13}$ In order to avoid postoperative neurological deficits, the use of intrasurgical electrical mapping during awake craniotomy has increased during the past 20 years. This intervention has been shown to increase the EOR, improve the quality of life postsurgery, and extend the OS in patients with supratentorial LGG, as well as allow for the mapping of language function intraoperatively. ${ }^{14}$ Adding intraoperative direct electrostimulation mapping allows real-time examinations of neural networks, which helps in evaluating sensorimotor, visuospatial, higher cognitive, and emotional functions. ${ }^{15}$

\section{Radiotherapy}

The role of radiotherapy in managing adult LGGs has long been controversial among neuro-oncologists. Previous studies have classified LGG patients into low- and high-risk groups based on their age and the EOR. One of these studies defined low-risk patients as those younger than 40 years of age and having undergone GTR as determined by the neurosurgeon, with high-risk patients being either 40 years or older or having received incomplete resection (STR or NTR). ${ }^{16}$ That study found that the 5 -year recurrence rate was $52 \%$ in low-risk patients, which supports the vital role of continuous MRI surveillance in detecting recurrence in these patients, who typically present only with seizures. ${ }^{16}$ Adjuvant therapy (radiotherapy and chemotherapy) can be deferred initially at the time of diagnosis in these low-risk patients who are in good condition, as long as they are monitored meticulously. ${ }^{17}$ A particularly perplexing subgroup of LGG patients do not harbor the favorable $I D H$ mutation (i.e., wild type) but are younger than 40 years and have undergone GTR. These patients could benefit from immediate postoperative concomitant radiotherapy and chemotherapy, since a 'wait-and-see' approach is not advised due to the predicted poor prognosis. ${ }^{18}$

Adjuvant therapy is strongly recommended in high-risk patients; that is, those 40 years or older or having undergone incomplete resections. ${ }^{18}$ There are two main decisions associated with the obligation to treat these patients with radiation: 1) the required dose of radiation and 2) the timing of administering radiation. Several randomized trials have addressed these challenges by comparing early (postresection) versus late (at progression) radiation in LGG patients. The currently applied dose of radiation, comprising 50-54 Gy given in fractions of $1.8 \mathrm{~Gy}$, was agreed upon after two randomized trials were implemented. The first was EORTC trial 22,844 , which was conducted in the 1980s and found no significant differences in OS and PFS between patients receiving $45 \mathrm{~Gy}$ of radiation over 5 weeks and those receiving $59.4 \mathrm{~Gy}$ over 6.6 weeks, with OS rates of $58 \%$ and 59\%, respectively, and PFS rates of $47 \%$ and $50 \%{ }^{19}$ The subsequent prospective 
trial performed in 2002 compared the therapeutic effects of low-dose (50.4 Gy) and high-dose (64.8 Gy) radiotherapy, and found that the OS rate was lower and the rate of radiation necrosis was slightly higher for high-dose radiation. These findings paved the way to the currently accepted dose range of $50-54 \mathrm{~Gy}^{4}{ }^{4}$

More importantly, EORTC trial 22,845, which was launched in 1986 to compare early postoperative radiotherapy with an observation-based 'wait-and-see' approach (i.e., radiation given at the time of progression), found that PFS increased slightly with no change in OS in LGG patients who received early radiotherapy as compared to late treatment following observation. ${ }^{20}$ Van den Bent et al. ${ }^{17}$ expanded on that trial with a 93-month follow-up, with their results also favoring the group treated with radiation immediately at diagnosis, as indicated by an increase in PFS (5.3 years vs. 3.4 years) and a better seizure control at a 1-year set point. However, OS was still indistinguishable between the two groups.

Despite the beneficial effects of radiation therapy in highrisk patients suffering from LGGs, this treatment has some significant side effects that should not be disregarded. The many studies that have addressed the cognitive risks of radiotherapy have used different scales to measure cognitive performance and impairment, one of which is the Mini Mental State Examination (MMSE) that comprises a 30item questionnaire. One of these studies found that patients with an abnormal baseline MMSE score before radiotherapy were more likely to experience improvement in their cognitive capacities following radiation treatment, ${ }^{21}$ although a small proportion of the studied patients showed cognitive decline after treatment. A similar study found that leukoencephalopathy and long-term memory decline were more severe in patients who had received radiotherapy postresection. ${ }^{22}$ These prominent side effects of radiotherapy should therefore be taken into consideration in a patient-specific manner when planning the management of LGGs. Continuing improvements in imaging techniques will help to prevent radiation-induced damage of normal tissue while continuing to target malignant areas. ${ }^{23}$

\section{Chemotherapy}

The optimal treatment for LGGs has yet to be established, and several treatment modalities are applied in practice. The risk-benefit ratio of radiation and chemotherapy should be determined for each individual patient while considering molecular markers. A patient with LGG that has undergone incomplete resection or is 40 years or older is considered a high-risk patient. Instead of the standard radiation treatment, adjuvant chemotherapy should be considered a better alternative for such high-risk patients. A large trial conducted by
Buckner et al. ${ }^{24}$ that included patients with high-risk LGGs found that patients who were treated with procarbazine, lomustine, and vincristine (PCV) after undergoing radiation therapy had a significantly longer median OS time (13.3 years) than those who received radiation alone (7.8 years). Moreover, the group that received both radiation and chemotherapy had a 10 -year PFS rate of $51 \%$, compared to $21 \%$ for the group that received radiation only. While the RTOG98-02 trial did not show any survival benefit of PCV combined therapy plus radiotherapy compared to radiotherapy alone in high-risk grade II astrocytomas, patients surviving beyond 2 years had improved PFS and OS when treated with combined chemotherapy and radiotherapy. ${ }^{25}$ Factors associated with improved survival in these patients were being younger than 40 years ( $51 \%$ vs. $20 \%, p=0.001$ ), receiving GTR or STR instead of biopsy only ( $56 \%$ vs. $33 \%, p=0.006$ ), and having either OD or OD-dominant mixed glioma histology (61\% vs. $35 \%$, $p=$ $0.003)^{25}$

After the recent subclassification of LGGs into three different molecular subtypes, studies have attempted to identify correlations between molecular diagnostic information and therapy outcomes. Although the trial of Buckner et al. ${ }^{24}$ was published after the WHO classification, it had begun 18 years prior, and so tumor tissue was unavailable for most patients, making it impossible to establish any such correlations. In order to guide therapeutic use, further studies are investigating the benefit of chemotherapy and radiation in association with different molecular subtypes. The studies performed thus far have shown that patients receiving PCV combined therapy displayed satisfactory results regardless of their $1 \mathrm{p} / 19 \mathrm{q}$ status. ${ }^{24,26,27}$ Patients with either anaplastic OD or low-grade OD tumors without either $1 \mathrm{p}$ or $19 \mathrm{q}$ deletion displayed good responses to PCV combined therapy when assessed for progression using MRI. However, patients without $1 \mathrm{p} / 19 \mathrm{q}$ codeletion exhibited median recurrence time of 18 months, which was shorter than the time for disease progression in any of the patients with $1 \mathrm{p} / 19 \mathrm{q}$ codeletion. ${ }^{27}$ Although the response duration appears to be shorter in patients without $1 \mathrm{p} / 19 \mathrm{q}$ codeletion, a response was nevertheless observed for both subtypes. However, due to the small sample size, further conclusions could not be drawn and additional studies must be conducted. Therefore, even patients without $1 \mathrm{p} / 19 \mathrm{q}$ codeletion - which is considered a less-favorable subtype than TP53 mutation-may respond to PCV combined therapy. Thus, the combination of radiation and chemotherapy is recommended for patients with high-risk LGGs at the initial diagnosis.

The PCV combined therapy in addition to radiotherapy was the preferred treatment choice for patients with newly diagnosed high-risk LGGs. ${ }^{24}$ However, temozolomide (TMZ) 
has recently been investigated as a potentially less-toxic alternative to PCV combined therapy. Similarly to PCV, TMZ is an oral alkylating agent that exhibits antitumor activity in the treatment of gliomas. ${ }^{28,29,30} \mathrm{TMZ}$ works by depleting the DNA-repair enzyme $\mathrm{O}^{6}$-alkylguanine-DNA alkyltransferase ${ }^{31}$ and methylating guanine and adenine, which together result in a continuous cycle of DNA base-mismatch repair leading to apoptosis. ${ }^{32}$ Preliminary results from an ongoing phase II study comparing adjuvant TMZ with concomitant radiation therapy to historical controls have been promising. ${ }^{33}$ Patients receiving adjuvant TMZ have shown a $20 \%$ improvement in the 3 -year OS rate (from 54\% to 65\%), which further supports TMZ treatment for high-risk LGGs. While the median survival times have not yet been reached, a recent study involving volumetric analysis of the LGG subgroup with the best prognosis (1p/19q-codeleted LGGs) showed that TMZ may have a less-prolonged effect on volume control than PCV combined therapy. ${ }^{32}$ The NCT00887146 ongoing phase III trial entitled 'Radiation Therapy With Concomitant and Adjuvant Temozolomide Versus Radiation Therapy With Adjuvant PCV Chemotherapy in Patients With Anaplastic Glioma or Low Grade Glioma' is aiming to compare the PFS, toxicity, and neurocognitive effects between TMZ and PCV combined therapy in newly diagnosed 1p/19q-codeleted highrisk LGGs. That study will hopefully yield more comparative insight into using TMZ versus the current PCV combined therapy in this subgroup. While the OS remained comparable, the NOA-04 trial of anaplastic glioma patients by Wick et al. ${ }^{34}$ showed less toxicity in the TMZ arm than for PCV combined therapy, in terms of allergic reactions (1\% vs. 19\%), hematological toxicities (4\% vs. $21 \%$ ), and polyneuropathy (0\% vs. $15 \%)$. Similarly, the RTOG9813 trial compared patients receiving radiotherapy and TMZ versus carmustine/lomustine, and found higher toxicity and a lower rate of chemotherapy completion in the latter, while maintaining comparable median OS times ( 3.9 vs. 3.8 years). ${ }^{35}$

The most-common side effects of TMZ include fatigue, lymphopenia, leukopenia, constipation, and nausea. ${ }^{36,37}$ A retrospective review of 25 patients with LGGs treated with TMZ showed no grade- 5 toxicity (death), $48 \%$ grade- 3 lymphopenia, and no grade- 4 lymphopenia. ${ }^{37}$ One of the difficulties associated with using TMZ is the emergence of resistance after prolonged treatment, which prevents the application of multiple cycles of TMZ. This resistance is mainly due to upregulation of DNA repair enzyme methylguanine-DNA methyltransferase (MGMT). MGMT works by repairing the TMZinduced $\mathrm{O}^{6}$-alkylguanine DNA adducts, and therefore negates its therapeutic cytotoxic effects. ${ }^{38}$ Since the methyl group transfer reaction to MGMT is a suicidal one, a possible method to counteract this therapeutic challenge would be to administer
DNA adducts at the $\mathrm{O}^{6}$ position. This could completely deplete MGMT levels, and the rate of DNA alkylation would theoretically outpace the rate of MGMT synthesis. Potent MGMT inhibitors include $\mathrm{O}^{6}$-benzylguanine and $\mathrm{O}^{6}$-(4-bromothenyl) guanine (PaTrin-2). ${ }^{38}$ More specifically, an increased TMZ response was also associated with $1 \mathrm{p}$ deletion and low MGMT expression..$^{39,40}$ Levin et al. ${ }^{40}$ demonstrated this in patients with progressive low-grade OD who were treated with TMZ. After analyzing the $1 \mathrm{p} / 19 \mathrm{q}$ status and estimating MGMT protein expression, MRI was used to evaluate clinical outcomes. That study demonstrated that patients with the highest MGMT levels had no deletion of 1p, while patients with $1 \mathrm{p}$ deletion tended to exhibit the lowest expressions of MGMT. Moreover, significant correlations were found between the radiographic response to $\mathrm{TMZ}, 1 \mathrm{p}$ deletion, and low MGMT levels. These observations support using 1p deletion or even MGMT immunostaining as markers to predict the chemosensitivity to $\mathrm{TMZ}$ and guide its therapeutic usage. The deletion of $1 p$ is correlated with the radiographic response and could be a helpful marker for guiding therapeutic decision-making in LGG. However, while the aforementioned factors can show favorable responses, TMZ therapy was found to exert an unfavorable effect in recurrent LGG, with tumors acquiring a hypermutation phenotype that could lead to malignant transformation. ${ }^{41}$ Additional studies are therefore needed to ensure that TMZ is given only to those patients who could potentially benefit from it.

The use of chemotherapy alone has also been investigated in an attempt to avoid the side effects of radiation therapy. A substantial response rate has been found for TMZ in the treatment of high-risk LGGs in several phase II trials..$^{37,39,40,42}$ Brada et al. $^{42}$ assessed the efficacy of TMZ in patients with grade II gliomas who were treated surgically and had a median age of 40 years (age range of 25-68 years), and found 3-year PFS and OS rates of $66 \%$ and $82 \%$, respectively. Moreover, $54 \%$ of the patients with epilepsy exhibited a reduction in seizure frequency. A large phase III trial that compared treatment with radiotherapy to TMZ in high-risk LGGs (EORTC trials 22,033-26,033) found no significant difference in the PFS at a median follow-up of 48 months. ${ }^{43}$ That study also identified predictive molecular factors and confirmed that the PFS rates differed significantly between three subgroups: 1) IDH mutation with $1 \mathrm{p} / 19 \mathrm{q}$ codeletion, 2) IDH mutation without 1p/19q codeletion, and 3) wild-type IDH. Patients with an IDH mutation and no codeletion had a longer PFS when treated with radiotherapy ( 55 months) than with TMZ (36 months). However, there were no treatment-dependent differences for patients with combined IDH mutation and $1 \mathrm{p} / 19 \mathrm{q}$ codeletion and $I D H$ wild-type tumors. 


\section{The future of LGG therapy}

Some types of LGGs are capable of infiltrating very eloquent areas of the brain, which may make them inoperable. Such cases could be treated using stereotactic brachytherapy by placing an implantable irradiation source in the vicinity of the tumor, which is both effective and has a lower risk of complications. ${ }^{44}$ Novel therapies are currently being investigated to reduce the potentially life-threatening side effects of radiotherapy and chemotherapy that are mainly due to the inadvertent killing of normal cells while targeting malignant cells. One way to avoid targeting normal cells is to inhibit specific pathways that are increasingly activated in LGG cancer cells, such as the PI3K/AKT/mTOR pathway. ${ }^{45}$ Everolimus is an inhibitor of the mTOR pathway that is currently being applied to patients with recurrent LGGs, and has produced positive results in terms of increased disease stability ${ }^{46}$ The unfavorable outcomes for both PFS and OS in this patient population are due to activation of the PI3K/mTOR pathway (as detected using immunostaining for $\mathrm{p}$-S6). A phase I multicenter RCT (NCT03343197) is currently being designed for studying the therapeutic benefits of AG-120 and AG-881 in patients with $I D H$-mutant LGGs. These drugs specifically inhibit the mutated form of IDH, and the effect of administering them preoperatively will be examined in resected tumors by measuring the suppression of 2-hydroxyglutarate, which is an oncometabolite of mutated IDH.

Immunotherapy employs the patient's own immune system to selectively attack cancer cells, and this is another appealing antitumor therapy technique that has been receiving increasing attention. A recent systematic review demonstrated that both active and passive immunotherapy greatly increased the OS and PFS in glioma patients, although no distinction was made between LGG and HGG. ${ }^{47}$ An exciting subtype of immunotherapy is vaccine therapy, of which one type utilizes dendritic immune cells that are cocultured with tumor cells from the patient, and thus targeting these cancer cells in vitro. ${ }^{48}$ Autologous dendritic cells pulsed with tumor lysate are then injected intradermally into the patient, which elicits an antigen-specific T-cell-mediated antitumor response. A major trial that is currently being conducted (NCT01635283) is investigating the role of these dendritic vaccines in the treatment of LGGs. Another current clinical trial (NCT02924038) is examining the role of varlilumab, a monoclonal antibody that targets the CD27 that activates lymphocytes against tumor cells, in combination with the IMA950 vaccine (containing 11 glioblastoma tumor-associated peptides) and poly-ICLC (an immunostimulant that might increase the tumoricidal effects of certain immunohematopoietic cells). That trial has shown that when IMA950 tumor peptides are administered to LGG patients, varlilumab amplifies the rate and magnitude of CD4+ and CD8+ T-cell responses directed against these IMA950 peptides, which is a very promising discovery.

In vivo studies of astrocytoma formation have shown that constitutively activated NTRK2 alleles, namely QKI-NTRK2 fusion, synergize with $I n k 4 a / A r f$ loss to promote the formation of an astrocytoma. This suggests that patients harboring astrocytomas with QKI-NTRK2 fusion could benefit from targeted therapies against NTRK2. ${ }^{49}$

An analysis of the TCGA study revealed several biologically discrete subsets and pathways of progression in diffuse glioma, including WHO grade II gliomas. ${ }^{50}$ Although the classification was made prior to the 2016 WHO classification, the grade II gliomas could be categorized into three major subtypes: G-CIMP high (IDH mutant), codeletion (IDH mutant), and pilocytic astrocytoma-like (IDH wild-type). While not yet implicated in clinical trials, this subclassification might further shed light on targeted therapies and the prognosis of patients with grade II gliomas.

\section{CONCLUSIONS}

The highly infiltrative nature of LGGs has made managing patients with this type of cancer difficult for decades. Clinical trials designed to investigate the different treatment modalities-including surgery, radiotherapy, and chemotherapy, and their combinations-have allowed researchers to begin to understand the mechanisms by which these tumors respond to treatment.

Low-risk patients are advised to undergo tumor resection combined with ongoing MRI-based monitoring to detect recurrence. On the other hand, high-risk patients can benefit from surgery with adjuvant therapy comprising a combination of radiotherapy and chemotherapy. Despite the benefits of surgery, radiotherapy, and chemotherapy, the highly invasive nature of LGGs can make them resistant to these conventional therapy modalities. Furthermore, these tumors eventually transform into HGGs, ultimately resulting in patient death. Like all other therapeutic interventions, it is essential to make individualized decisions while considering the trade-off between risks and benefits. The future of LGG therapy promises the specific targeting of LGGs while minimally affecting normal brain tissue, whether via inhibiting particular pathways that are increasingly activated in these tumors or by designing antitumor vaccines that augment the body's own immune response against this ominous type of slow-growing cancer.

The main gaps in current studies are related to the lack of certain specialized therapies-especially monoclonal antibodies-targeted to LGGs rather than strictly to high-grade glioblastomas. Future therapies will not only selectively attack 
cancer cells but may also enhance the quality of life of these patients, a factor that has not been studied extensively in the past due to a focus on treating the underlying pathology. Such better outcomes might be achievable as medicine evolves toward more-personalized therapies.

\section{Conflicts of Interest}

The authors have no financial conflicts of interest.

\section{REFERENCES}

1. Louis DN, Perry A, Reifenberger G, von Deimling A, FigarellaBranger D, Cavenee WK, et al. The 2016 World Health Organization classification of tumors of the central nervous system: a summary. Acta Neuropathol 2016;131:803-820.

2. Cancer Genome Atlas Research Network, Brat DJ, Verhaak RG, Aldape KD, Yung WK, Salama SR, et al. Comprehensive, integrative genomic analysis of diffuse lower-grade gliomas. N Engl J Med 2015;372: 2481-2498.

3. Ostrom QT, Gittleman H, Fulop J, Liu M, Blanda R, Kromer C, et al. CBTRUS statistical report: primary brain and central nervous system tumors diagnosed in the United States in 2008-2012. Neuro Oncol 2015;17 Suppl 4:iv1-iv62.

4. Shaw E, Arusell R, Scheithauer B, O'Fallon J, O'Neill B, Dinapoli R, et al. Prospective randomized trial of low- versus high-dose radiation therapy in adults with supratentorial low-grade glioma: initial report of a North Central Cancer Treatment Group/Radiation Therapy Oncology Group/Eastern Cooperative Oncology Group study. $J$ Clin Oncol 2002;20:2267-2276.

5. Bogdańska MU, Bodnar M, Piotrowska MJ, Murek M, Schucht P, Beck J, et al. A mathematical model describes the malignant transformation of low grade gliomas: prognostic implications. PLoS One 2017;12:e0179999.

6. Schiff D. Low-grade gliomas. Continuum (Minneap Minn) 2015;21: 345-354.

7. Capelle L, Fontaine D, Mandonnet E, Taillandier L, Golmard JL, Bauchet L, et al. Spontaneous and therapeutic prognostic factors in adult hemispheric World Health Organization grade II gliomas: a series of 1097 cases: clinical article. J Neurosurg 2013;118:1157-1168.

8. Smith JS, Chang EF, Lamborn KR, Chang SM, Prados MD, Cha S, et al. Role of extent of resection in the long-term outcome of low-grade hemispheric gliomas. J Clin Oncol 2008;26:1338-1345.

9. McGirt MJ, Chaichana KL, Attenello FJ, Weingart JD, Than K, Burger PC, et al. Extent of surgical resection is independently associated with survival in patients with hemispheric infiltrating low-grade gliomas. Neurosurgery 2008;63:700-708.

10. Cordier D, Gozé C, Schädelin S, Rigau V, Mariani L, Duffau H. A better surgical resectability of WHO grade II gliomas is independent of favorable molecular markers. J Neurooncol 2015;121:185-193.

11. Wijnenga MM, French PJ, Dubbink HJ, Dinjens WN, Atmodimedjo PN, Kros JM, et al. The impact of surgery in molecularly defined low-grade glioma: an integrated clinical, radiological, and molecular analysis. Neuro Oncol 2018;20:103-112.

12. Nimsky C, Fujita A, Ganslandt O, von Keller B, Fahlbusch R. Volumetric assessment of glioma removal by intraoperative high-field magnetic resonance imaging. Neurosurgery 2004;55:358-370; discussion $370-371$.

13. Claus EB, Horlacher A, Hsu L, Schwartz RB, Dello-Iacono D, Talos F, et al. Survival rates in patients with low-grade glioma after intraoperative magnetic resonance image guidance. Cancer 2005;103:12271233.

14. Duffau H. Is non-awake surgery for supratentorial adult low-grade glioma treatment still feasible? Neurosurg Rev 2018;41:133-139.
15. Duffau H. Mapping the connectome in awake surgery for gliomas: an update. J Neurosurg Sci 2017;61:612-630.

16. Shaw EG, Berkey B, Coons SW, Bullard D, Brachman D, Buckner JC, et al. Recurrence following neurosurgeon-determined gross-total resection of adult supratentorial low-grade glioma: results of a prospective clinical trial. J Neurosurg 2008;109:835-841.

17. van den Bent MJ, Afra D, de Witte O, Ben Hassel M, Schraub S, Hoang-Xuan $\mathrm{K}$, et al. Long-term efficacy of early versus delayed radiotherapy for low-grade astrocytoma and oligodendroglioma in adults: the EORTC 22845 randomised trial. Lancet 2005;366:985-990.

18. Oberheim Bush NA, Chang S. Treatment strategies for low-grade glioma in adults. J Oncol Pract 2016;12:1235-1241.

19. Karim AB, Maat B, Hatlevoll R, Menten J, Rutten EH, Thomas DG, et al. A randomized trial on dose-response in radiation therapy of lowgrade cerebral glioma: European Organization for Research and Treatment of Cancer (EORTC) Study 22844. Int J Radiat Oncol Biol Phys 1996;36:549-556.

20. Karim AB, Afra D, Cornu P, Bleehan N, Schraub S, De Witte O, et al. Randomized trial on the efficacy of radiotherapy for cerebral lowgrade glioma in the adult: European Organization for Research and Treatment of Cancer Study 22845 with the Medical Research Council study BRO4: an interim analysis. Int J Radiat Oncol Biol Phys 2002;52: 316-324.

21. Brown PD, Buckner JC, O’Fallon JR, Iturria NL, Brown CA, O'Neill $\mathrm{BP}$, et al. Effects of radiotherapy on cognitive function in patients with low-grade glioma measured by the folstein mini-mental state examination. J Clin Oncol 2003;21:2519-2524.

22. Surma-aho O, Niemelä M, Vilkki J, Kouri M, Brander A, Salonen O, et al. Adverse long-term effects of brain radiotherapy in adult lowgrade glioma patients. Neurology 2001;56:1285-1290.

23. Baumert BG, Stupp R. Is there a place for radiotherapy in low-grade gliomas? Adv Tech Stand Neurosurg 2010;35:159-182.

24. Buckner JC, Chakravarti A, Curran WJ Jr. Radiation plus chemotherapy in low-grade glioma. N Engl J Med 2016;375:490-491.

25. Shaw EG, Wang M, Coons SW, Brachman DG, Buckner JC, Stelzer KJ, et al. Randomized trial of radiation therapy plus procarbazine, lomustine, and vincristine chemotherapy for supratentorial adult low-grade glioma: initial results of RTOG 9802. J Clin Oncol 2012;30:3065-3070.

26. Buckner JC, Gesme D Jr, O’Fallon JR, Hammack JE, Stafford S, Brown $\mathrm{PD}$, et al. Phase II trial of procarbazine, lomustine, and vincristine as initial therapy for patients with low-grade oligodendroglioma or oligoastrocytoma: efficacy and associations with chromosomal abnormalities. J Clin Oncol 2003;21:251-255.

27. Stege EM, Kros JM, de Bruin HG, Enting RH, van Heuvel I, Looijenga LH, et al. Successful treatment of low-grade oligodendroglial tumors with a chemotherapy regimen of procarbazine, lomustine, and vincristine. Cancer 2005;103:802-809.

28. Newlands ES, Stevens MF, Wedge SR, Wheelhouse RT, Brock C. Temozolomide: a review of its discovery, chemical properties, pre-clinical development and clinical trials. Cancer Treat Rev 1997;23:35-61.

29. Stupp R, Gander M, Leyvraz S, Newlands E. Current and future developments in the use of temozolomide for the treatment of brain tumours. Lancet Oncol 2001;2:552-560.

30. Stupp R, Mason WP, van den Bent MJ, Weller M, Fisher B, Taphoorn $\mathrm{MJ}$, et al. Radiotherapy plus concomitant and adjuvant temozolomide for glioblastoma. N Engl J Med 2005;352:987-996.

31. Tolcher AW, Gerson SL, Denis L, Geyer C, Hammond LA, Patnaik A, et al. Marked inactivation of $\mathrm{O}^{6}$-alkylguanine-DNA alkyltransferase activity with protracted temozolomide schedules. Br J Cancer 2003;88: 1004-1011.

32. Izquierdo C, Alentorn A, Idbaih A, Simó M, Kaloshi G, Ricard D, et al. Long-term impact of temozolomide on $1 \mathrm{p} / 19 \mathrm{q}$-codeleted low-grade glioma growth kinetics. J Neurooncol 2018;136:533-539.

33. Fisher BJ, Hu C, Macdonald DR, Lesser GJ, Coons SW, Brachman DG, et al. Phase 2 study of temozolomide-based chemoradiation therapy 
for high-risk low-grade gliomas: preliminary results of Radiation Therapy Oncology Group 0424. Int J Radiat Oncol Biol Phys 2015;91: 497-504.

34. Wick W, Hartmann C, Engel C, Stoffels M, Felsberg J, Stockhammer F, et al. NOA-04 randomized phase III trial of sequential radiochemotherapy of anaplastic glioma with procarbazine, lomustine, and vincristine or temozolomide. J Clin Oncol 2009;27:5874-5880.

35. Chang S, Zhang P, Cairncross JG, Gilbert MR, Bahary JP, Dolinskas $\mathrm{CA}$, et al. Phase III randomized study of radiation and temozolomide versus radiation and nitrosourea therapy for anaplastic astrocytoma: results of NRG Oncology RTOG 9813. Neuro Oncol 2017;19:252-258.

36. Kesari S, Schiff D, Drappatz J, LaFrankie D, Doherty L, Macklin EA, et al. Phase II study of protracted daily temozolomide for low-grade gliomas in adults. Clin Cancer Res 2009;15:330-337.

37. Pouratian N, Gasco J, Sherman JH, Shaffrey ME, Schiff D. Toxicity and efficacy of protracted low dose temozolomide for the treatment of low grade gliomas. J Neurooncol 2007;82:281-288.

38. Fan CH, Liu WL, Cao H, Wen C, Chen L, Jiang G. $\mathrm{O}^{6}$-methylguanine DNA methyltransferase as a promising target for the treatment of temozolomide-resistant gliomas. Cell Death Dis 2013;4:e876.

39. Hoang-Xuan K, Capelle L, Kujas M, Taillibert S, Duffau H, Lejeune J, et al. Temozolomide as initial treatment for adults with low-grade oligodendrogliomas or oligoastrocytomas and correlation with chromosome 1p deletions. J Clin Oncol 2004;22:3133-3138.

40. Levin N, Lavon I, Zelikovitsh B, Fuchs D, Bokstein F, Fellig Y, et al. Progressive low-grade oligodendrogliomas: response to temozolomide and correlation between genetic profile and $\mathrm{O}^{6}$-methylguanine DNA methyltransferase protein expression. Cancer 2006;106:17591765.

41. Johnson BE, Mazor T, Hong C, Barnes M, Aihara K, McLean CY, et al. Mutational analysis reveals the origin and therapy-driven evolution of recurrent glioma. Science 2014;343:189-193.
42. Brada M, Viviers L, Abson C, Hines F, Britton J, Ashley S, et al. Phase II study of primary temozolomide chemotherapy in patients with WHO grade II gliomas. Ann Oncol 2003;14:1715-1721.

43. Baumert BG, Hegi ME, van den Bent MJ, von Deimling A, Gorlia T, Hoang-Xuan K, et al. Temozolomide chemotherapy versus radiotherapy in high-risk low-grade glioma (EORTC 22033-26033): a randomised, open-label, phase 3 intergroup study. Lancet Oncol 2016;17: 1521-1532.

44. Ruge MI, Kickingereder P, Grau S, Dorn F, Galldiks N, Treuer H, et al. Stereotactic iodine-125 brachytherapy for the treatment of WHO grades II and III gliomas located in the central sulcus region. Neuro Oncol 2013;15:1721-1731.

45. McCubrey JA, Steelman LS, Chappell WH, Abrams SL, Montalto G, Cervello M, et al. Mutations and deregulation of Ras/Raf/MEK/ERK and PI3K/PTEN/Akt/mTOR cascades which alter therapy response. Oncotarget 2012;3:954-987.

46. Wahl M, Chang SM, Phillips JJ, Molinaro AM, Costello JF, Mazor T, et al. Probing the phosphatidylinositol 3-kinase/mammalian target of rapamycin pathway in gliomas: a phase 2 study of everolimus for recurrent adult low-grade gliomas. Cancer 2017;123:4631-4639.

47. Hanaei S, Afshari K, Hirbod-Mobarakeh A, Mohajer B, Amir Dastmalchi D, Rezaei N. Therapeutic efficacy of specific immunotherapy for glioma: a systematic review and meta-analysis. Rev Neurosci 2018; 29:443-461.

48. Kim W, Liau LM. Dendritic cell vaccines for brain tumors. Neurosurg Clin N Am 2010;21:139-157.

49. Ni J, Xie S, Ramkissoon SH, Luu V, Sun Y, Bandopadhayay P, et al. Tyrosine receptor kinase B is a drug target in astrocytomas. Neuro Oncol 2017;19:22-30.

50. Ceccarelli M, Barthel FP, Malta TM, Sabedot TS, Salama SR, Murray BA, et al. Molecular profiling reveals biologically discrete subsets and pathways of progression in diffuse glioma. Cell 2016;164:550-563. 